\title{
Aberrant Thyroid in the Parapharyngeal Space
}

\author{
Sharanabasappa Rudragouda Malipatil, ${ }^{1}$ Ciju K George, $^{2}$ Isha Vidisha, ${ }^{3}$ Ravindrakumar Ningappa Karadi ${ }^{1}$
}

\section{Introduction:}

\section{ABSTRACT}

Aberrant thyroid is a mass of tissue having the structure of a normal or pathological thyroid gland and situated at some definite distance from normal thyroid, with which it has no connection. The prevalance of ectopic thyroid is 1 case per 100,000-300,000 persons 1 and the incidence of aberrant thyroid in parapharyngeal space is even rarer.

Materials and Methods

A rare case of aberrant thyroid in parapharyngeal space is reported, which presented as oropharyngeal mass and dysphagia.

She also had thyroid gland in the usual position.

$\underline{\text { Result }}$

She was managed surgically with transcervical approach and was euthyroid postoperatively.

$\underline{\text { Conclusion }}$

Aberrant thyroid should be one of the differential diagnoses for parapharyngeal space masses.

Kevwords:

Thyroid Dysgenesis; Neck

$\mathrm{P}$ arapharyngeal space is a potential space and the lesions here usually arise from anatomical structures within. The pre-styloidspace lesions frequently arise from salivary gland and almost always arise from the deep lobe of parotid.

The post-styloid space lesions are commonly neurogenic in origin and the most common ones are paraganglioma, schwannoma and neurofibroma. Aberrant means "wandering," and thyroid tissue anywherein the body outside the thyroid gland can becalled aberrant. We present an unusual case of parapharyngeal lesion, i.e. an aberrant thyroid in the presence of a normal functioning thyroid gland, which is extremely rare.

1 - Department of ENT, Shri B.M.Patil Medical College Hospital and Research Centre, Karnataka

2 - Department of ENT, P K Das institute of Medical

Sciences, Vaniamkukam, Palakkad, Kerala

3 - ENT Consultant, Plot Number -2656/3293

Sabara Sahi, Biswanath Nagar, Bhubaneshwar

\section{Corresponding author:}

Dr Ciju K George

email: cijukg@yahoo.co.in

\section{Case report}

A35-year-old female presented with a slowly progressive, painless swelling in the oropharynx since two years. There was associated bilateral nasal obstruction, more in the right side and dysphagia since last one year. There was no history of heat intolerance, palpitations, excessive sweating or anxiety. There was no family history of thyroid disorders or malignancies. On examination, she was comfortable, and no stridor was observed. There was a firm, non tendermass in the oropharynx pushing the right tonsil medially and extending into posterior pharyngeal wall (Fig. 1). There was no obvious swelling in the neck.

Blood investigations, which included a full blood count, renalprofile and random blood glucose, were normal.Magnetic resonance (MR) imaging of the neck and thoraxshowed a heterogenous mass with few cystic areas, fat components and calcifications involving the right parapharyngeal space. The mass measured $50 \mathrm{x}$ $30 \times 75 \mathrm{~mm}$ extending from clivus to C5 vertebral body, obliterating the nasopharynx and extending upto the the oropharynx (Fig. 2). The leftparapharyngeal space was clear. It also showed thyroid in the normal site with few 


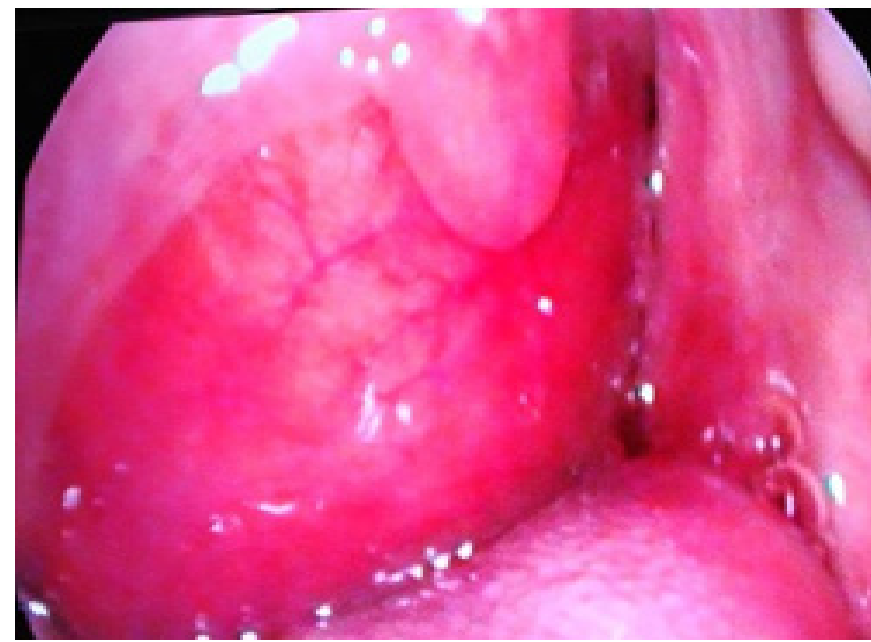

Fig 1.Right parapharyngeal mass causing bulge in the oropharynx

specks of coarse calcification and few hypodense areas. Following admission, fine-needle aspiration cytology was performed. Histology showed mainly thyroid follicular cells arranged in mononuclear sheets, clusters and in follicles with background of hemosiderin laded macrophages. The thyroid function test performed preoperatively was within normal range.

A transcervical excision of the tumourwas performed to remove the tumour in toto from the right parapharyngeal space (Fig. 3). Jugulo-digastric lymph node was sampled. Histologicaldiagnosis of the tumour mass showed normal thyroid follicles lined by cuboidal

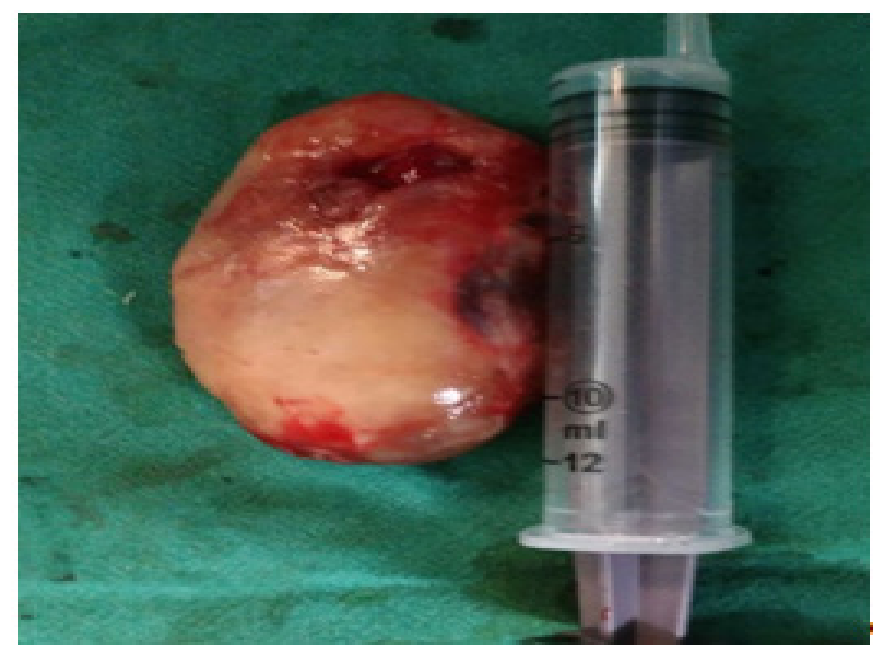

Fig. 3.Surgical specimen of the parapharyngeal mass

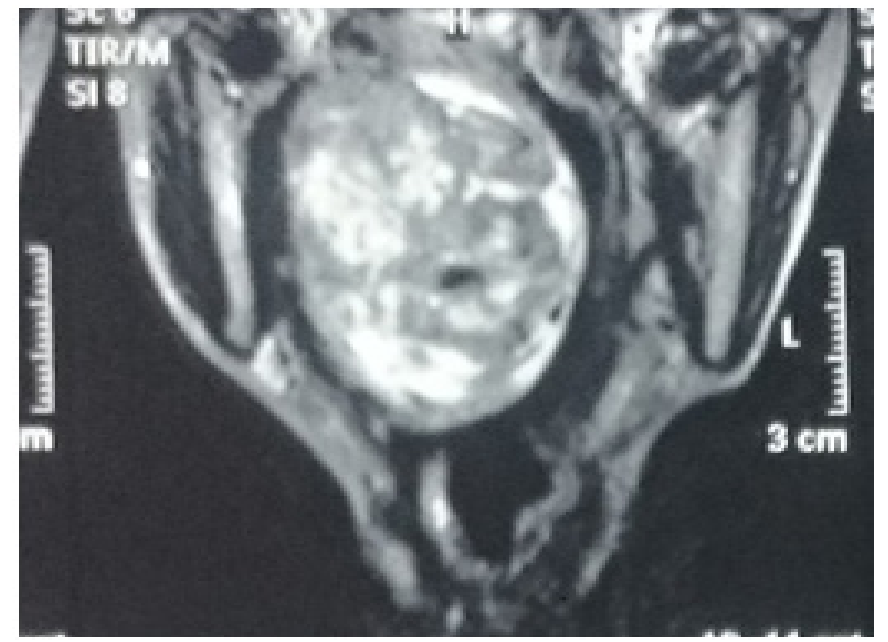

Fig 2. MRI showing right parapharyngeal mass

epithelium and filled with colloid. There was no evidence of malignancy. The enlarged lymph nodesshowed reactive lymphadenitis. Postoperativethyroid function, after one week of surgery was within normal limits.

\section{Discussion}

Thyroid follicular cells are derived from both a median thyroid and a lateral thyroid bud. These lateral thyroid anlagens are derived from the ultimo-branchial body, a descending diverticulum of the fourth pharyngeal pouch. ${ }^{1}$ Aberrant thyroid tissues found in the lateral

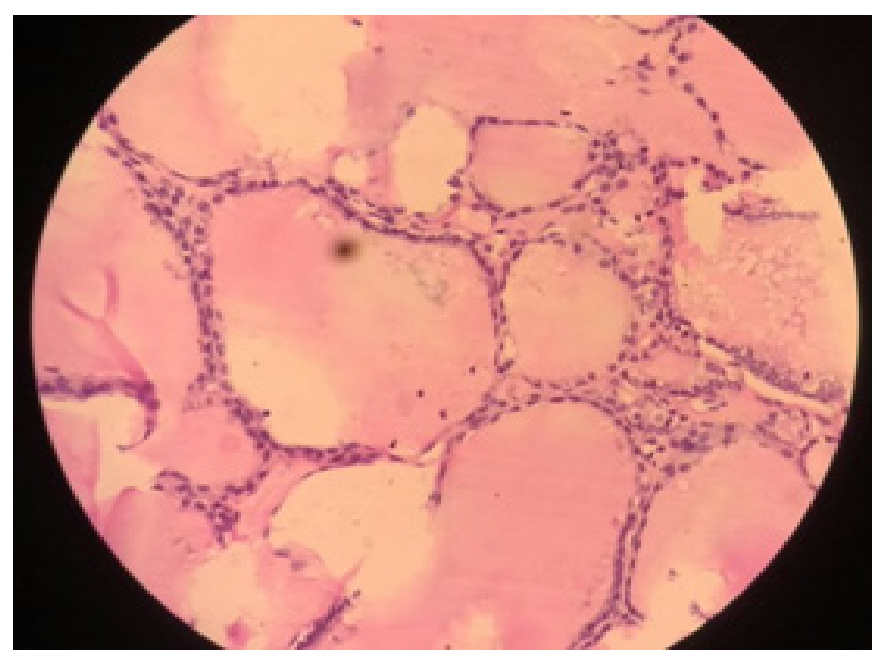

Fig. 4. Thyroid follicles filled with colloid (H\&E, 40x) 
neck regions could originate from defective lateral thyroid components, which fail to migrate and fuse with the median thyroid anlage. ${ }^{2}$ This failure could lead to the ectopia of the lateral anlage in such unusual sites like parapharyngeal space. The existence of the lateral thyroid anlagen has been a topic of controversy, but its existence may explain the occurrence of non-midline ectopic thyroid tissue in the neck. ${ }^{3}$ Many researchers have described that gene of transcription factorsTITF1(Nkx2-1), Foxe1(TITF-2) and PAX-8 are essential for thyroid morphogenesis and differentiation. ${ }^{4,5}$ Mutation in these genes may be involved in abnormal migration of thet hyroid resulting in ectopic and aberrant thyroid. ${ }^{5}$

These lateral aberrant groups of cells which fail to meet the thyroid proper later become activated and may give rise to tumours. Seventy percent of these aberrant thyroids give rise to neoplasms of papillary type. The other lesions are papillary adenocarciinoma, epithelioma, and alveolar carcinoma. The tumor is usually slow-growing and subject to involutional changes such as cystic degeneration hemorrhage and calcification. A well-defined capsule is usually present. They may undergo malignant changes and give rise to metastases. ${ }^{6}$ Such cases of lateral aberrant thyroid malignancies were previously reported by Johnson and Saha. ${ }^{7}$ To our knowledge, we are aware of only four published reports of ectopic thyroid inthe pharynx. ${ }^{8-11}$

This patient had an ectopic parapharyngeal thyroid gland in the presence of a normal functioning thyroid. Her main presenting complaint had been a mass in the oropharynx, which was slowly increasing in size over two years. She also developed bilateral nasal obstruction and dysphagia since one year. Following complete excision, no further intervention was planned as the tumour was proved to be benign thyroid tissue by histopathological examination and she was euthyroid on subsequent thyroid function tests. It is important to exclude malignancy because a differential diagnosis of lateral aberrant thyroid is metastasis from a primary thyroid carcinoma. ${ }^{12}$ The treatment of ectopic thyroid depends on its location, size and on the presence of symptoms or complications. For a nonfunctioning ectopic thyroid in the presence of a normal thyroid gland, the indication for surgery depends on the patient's symptoms. Surgical excision was warranted in this patientas the mass effect by the tumour caused nasal obstruction and dysphagia. The transcervical approach to the tumour in this case allowed the surgeona wider access to post styloid compartment of parapharyngeal region for complete excision of the tumour mass. The surgical team was ready for an extended transmandibular approach if needed. Postoperatively, the histopathology reported no evidence of malignancy and the patientwas biochemically euthyroid, thus rendering no further intervension. Thus, an aberrant thyroid should be considered in the differential diagnoses of parapharyngeal neoplasm.

\section{Conclusion}

Aberrant thyroid in the parapharyngeal space is a rare disease. Although the cause is not fully known, genetic factors is thought to play an important role in such cases. The majority are asymptomatic; however, symptoms related to tumor size and location may develop. MRI imaging, Fine Needle Aspiration cytology and thyroid function tests are the main diagnostic tools. Surgical excision is the treatment of choice in symptomatic cases, with a role for radioiodine ablation only in recurrent disease. The clinician should always take into account the potential of this rare entity while dealing with parapharyngeal masses.

\section{References}

1. Guerra G, Cinelli M, Mesolella M, Tafuri D, Rocca A, Amato B et al. Morphological, diagnostic and surgical features of ectopic thyroid gland: A review of literature. International Journal of Surgery 2014; 12:S3-S11

2. Misaki T, Koh T, Shimbo S, Kasagi K, Konishi J. Dual-Site Thyroid Ectopy in a Mother and Son. Thyroid 1992; 2(4):325-7

3. Gray H, Williams PL, ed. Gray's Anatomy: The Anatomical Basis of Medicine and Surgery. 38th ed. London: Churchill Livingstone, 1995

4. Szinnai G. Genetics of normal and abnormal thyroid development in humans, Best. Pract. Res. Clin. Endocrinol. Metabolism 2014; 28(2)133e150

5. Gillam MP, Kopp P. Genetic regulation of thyroid development, Curr. Opin. Pediatr. 2001; 13: 358e363

6. Lazarus JA, Rosenthal AA. Lateral aberrant thyroid glands. 
Annals of Surgery 1933;98(6): 1023-9

7. Johnson R, Saha N. The So-called Lateral Aberrant Thyroid. BMJ 1962;1(5293):1668-9

8. Soscia A, Guerra G, Cinelli MP, et al. Parapharyngeal ectopic thyroid: the possible persistence of the lateral thyroid anlage. Clinical case report. Surg Radiol Anat. 2004; 26:338-43

9. Basak S, Basak O, Odabasi O, Düzcan E. Pharyngeal thyroid: A case report. Br J Oral Maxillofac Surg. 1999; 37:61-3
10. Shvartsman AIa, TolchinskiI VV, Meshcheriakova IuIa. Case of an aberrant thyroid gland in the peripharyngeal space. Vestn Otorinolaringol. 1978:66-7

11. 11.Feisal T K T, Prepageran N, Shahrizal T, Zulkiflee A B. Unsual parapharyngeal lesion: aberrant thyroid gland. Singapore Med J. 2008; 49(5):e137

12. Clay RC, Blackman SS Jr. Lateral aberrant thyroid: metastasis to lymph nodes from primary carcinoma of the thyroid gland. Arch Surg. 1944; 48:223-8. 